\title{
THE CLASSIFICATION \\ OF TWO-COMPONENT CUNTZ-KRIEGER ALGEBRAS
}

\author{
DANRUN HUANG
}

(Communicated by Palle E. T. Jorgensen)

\begin{abstract}
Cuntz-Krieger algebras with exactly one nontrivial closed ideal are classified up to stable isomorphism by the Cuntz invariant. The proof relies on Rørdam's classification of simple Cuntz-Krieger algebras up to stable isomorphism and the author's classification of two-component reducible topological Markov chains up to flow equivalence.
\end{abstract}

\section{Preliminaries}

Let $A \in \mathrm{M}_{n}(\{0,1\})$ be nondegenerate (no zero rows or columns) such that no irreducible component is a permutation matrix. The Cuntz-Krieger $C^{*}$-algebra (CK-algebra) $\mathcal{O}_{A}$ associated to $A$ is defined in [CK] as the $C^{*}$-algebra generated by partial isometries $s_{1}, s_{2}, \ldots, s_{n}$, satisfying the relations

$$
\begin{gathered}
1=s_{1} s_{1}^{*}+s_{2} s_{2}^{*}+\cdots+s_{n} s_{n}^{*} \\
s_{i}^{*} s_{i}=\sum_{j=1}^{n} A(i, j) s_{j} s_{j}^{*}
\end{gathered}
$$

The assumption ruling out permutation components is needed to guarantee that $\mathcal{O}_{A}$ is uniquely defined up to isomorphism [CK], [C1].

Cuntz showed in $[\mathrm{C} 1]$ that there is a bijective correspondence between the closed ideals of $\mathcal{O}_{A}$ and the hereditary subsets of the poset $\Gamma_{A}$ of irreducible components of $A$. In particular, $\mathcal{O}_{A}$ is simple if and only if $A$ is irreducible, and $\mathcal{O}_{A}$ has exactly one nontrivial closed ideal if and only if $A$ is indecomposable (that is, $\Gamma_{A}$ is not a union of two order-disconnected proper subsets) and has exactly two irreducible components. In this note, we will discuss exclusively the latter case. For $A$ decomposable with two irreducible components, the associated algebra $\mathcal{O}_{A}$ is simply the direct sum of its two simple CK-subalgebras (ideals). In this case, the classification of two-component CK-algebras is trivially reduced to the classification of simple CK-algebras which has been accomplished just recently in [R],[C3].

Received by the editors June 13, 1994 and, in revised form, August 30, 1994.

1991 Mathematics Subject Classification. Primary 46L35, 54H20; Secondary 46L55.

Key words and phrases. Cuntz-Krieger algebra, stable isomorphism, topological Markov chain, flow equivalence. 
Given $A \in \mathrm{M}_{n}(\{0,1\})$, one can also define a topological Markov chain (i.e., shift of finite type) $\sigma_{A}$ associated to $A$. If $\Sigma$ is a set of $n$ symbols, then $\sigma_{A}$ is the shift homeomorphism of the compact space

$$
X_{A}=\left\{x=\left(x_{i}\right) \in \Sigma^{\mathbb{Z}} \mid A\left(x_{i}, x_{i+1}\right)=1\right\}
$$

defined by $\left(\sigma_{A} x\right)_{i}=x_{i+1}$. (See [Bo] for symbolic dynamics background and references.)

Two topological Markov chains (or their adjacency matrices) are said to be flow equivalent (FE) if their suspension flow spaces are homeomorphic under a homeomorphism that maps flow lines onto flow lines and preserves the orientation of the flow lines. Parry and Sullivan [PS] found matrix generators of flow equivalence and showed that $\operatorname{det}(I-A)$ is an invariant of flow equivalence. Later Bowen and Franks $[\mathrm{BF}]$ showed that the finitely generated abelian group

$$
B F(A):=\frac{\mathbb{Z}^{n}}{(I-A) \mathbb{Z}^{n}}
$$

is an invariant of flow equivalence, which we will call the Bowen-Franks group of $\sigma_{A}$ (or $A$ ). Franks showed in $[\mathrm{F}]$ that the above two invariants completely characterize flow equivalence of irreducible topological Markov chains whose adjacency matrices are not a permutation. Note that $B F(A)$ determines $\operatorname{det}(I-A)$ up to its sign: $0,+$, or - , which we will denote by $\operatorname{sgn}(A)$.

The stabilization of $\mathcal{O}_{A}$ is $\overline{\mathcal{O}}_{A}:=\mathcal{O}_{A} \otimes \mathcal{K}$, where $\mathcal{K}$ is the the algebra of all compact operators on a Hilbert space. $\mathcal{O}_{A}$ and $\mathcal{O}_{A^{\prime}}$ are said to be stably isomorphic, if $\overline{\mathcal{O}}_{A} \cong \overline{\mathcal{O}}_{A^{\prime}}$.

Theorem 1.1 (Cuntz-Krieger $[\mathrm{CK}],[\mathrm{C} 1]) . A \sim_{F E} A^{\prime}$ implies $\overline{\mathcal{O}}_{A} \cong \overline{\mathcal{O}}_{A^{\prime}}$. Moreover, $\operatorname{Ext}\left(\mathcal{O}_{A}\right) \cong B F(A), \quad K_{0}\left(\mathcal{O}_{A}\right) \cong B F\left(A^{t}\right)$ and $K_{1}\left(\mathcal{O}_{A}\right) \cong \operatorname{Ker}\left(I-A^{t}\right)$ (on $\left.\mathbb{Z}^{n}\right)$.

For $\mathcal{O}_{A}$ simple, Mikael Rørdam showed recently in $[\mathrm{R}]$ that $\overline{\mathcal{O}}_{A} \cong \overline{\mathcal{O}}_{A^{\prime}}$ if and only if $K_{0}\left(\mathcal{O}_{A}\right) \cong K_{0}\left(\mathcal{O}_{A^{\prime}}\right)$. On the other hand, it was shown in $[\mathrm{H}]$ that for a nonpermutation irreducible matrix $A$, any automorphism of $B F(A)$ can be realized by a flow equivalence $A \sim_{F E} A$. Using these new results, Cuntz showed that $\mathcal{O}_{A} \cong$ $\mathcal{O}_{A^{\prime}}$ (unital isomorphism) if and only if there is an isomorphism $\alpha_{0}: K_{0}\left(\mathcal{O}_{A}\right) \rightarrow$ $K_{0}\left(\mathcal{O}_{A^{\prime}}\right)$ such that $\alpha_{0}\left[1_{A}\right]=\left[1_{A^{\prime}}\right] \quad([\mathrm{R}],[\mathrm{C} 3])$. Thus the classification of simple CK-algebras, a problem that had been open for more than twelve years, was solved completely.

\section{Stable isomorphisms of TWO-COMPONENT CK-ALGEBRAS}

Cuntz [C1] began the study of nonsimple CK-algebras and flow equivalence of reducible topological Markov chains. He especially discussed the case in which $\mathcal{O}_{A}$ has exactly one nontrivial closed ideal. In that case, the indecomposable defining $\{0,1\}$-matrix $A$, modulo conjugation by a permutation matrix, has the block uppertriangular form

$$
A=\left(\begin{array}{cc}
A_{1} & X \\
0 & A_{2}
\end{array}\right)
$$


where the square submatrices $A_{1}$ and $A_{2}$ are essentially irreducible (that is, their maximum nondegenerate principal submatrices are irreducible) and neither irreducible component is a permutation. Some important properties of endomorphisms of simple $\mathcal{O}_{A}$ were proved by studying the corresponding two-component CK-algebras [C4].

Suppose $A_{1} \in M_{n}\left(\mathbb{Z}_{+}\right)$and $A_{2} \in M_{m}\left(\mathbb{Z}_{+}\right)$. Then $X$ can be identified with an element of $\mathbb{Z}^{n} \times \mathbb{Z}^{m}$ through

$$
X=\sum_{\substack{1 \leq i \leq n \\ 1 \leq j \leq m}} X(i j)\left(e_{i} \otimes f_{j}\right)
$$

where $e_{1}, \ldots, e_{n}$ and $f_{1}, \ldots, f_{m}$ are the standard bases of $\mathbb{Z}^{n}$ and $\mathbb{Z}^{m}$. Let $q_{1}$ : $\mathbb{Z}^{n} \rightarrow \mathbb{Z}^{n} /\left(I-A_{1}\right) \mathbb{Z}^{n}$ and $q_{2}: \mathbb{Z}^{m} \rightarrow \mathbb{Z}^{m} /\left(I-A_{2}^{t}\right) \mathbb{Z}^{m}$ be the quotient maps, then $X$ defines naturally an equivalence class $[X]:=\left(q_{1} \otimes q_{2}\right)(X) \in B F\left(A_{1}\right) \otimes B F\left(A_{2}^{t}\right)$.

Consider

$$
A=\left(\begin{array}{cc}
A_{1} & X \\
0 & A_{2}
\end{array}\right), \quad A^{\prime}=\left(\begin{array}{cc}
A_{1}^{\prime} & X^{\prime} \\
0 & A_{2}^{\prime}
\end{array}\right)
$$

Definition 2.1. Let $A, A^{\prime} \in \mathrm{M}_{k}\left(\mathbb{Z}_{+}\right)$be indecomposable with two irreducible components and having the forms as in (2-2), where $A_{1}, A_{2}, A_{1}^{\prime}$ and $A_{2}^{\prime}$ are essentially irreducible (but can be essential permutations). The Cuntz invariant $\mathcal{C}(A)$ of $A$ is the pair $\left([X], B F\left(A_{1}\right) \otimes B F\left(A_{2}^{t}\right)\right)$, modulo product-type isomorphisms. Precisely, we say $\mathcal{C}(A)=\mathcal{C}\left(A^{\prime}\right)$ if there exist isomorphisms $\delta: B F\left(A_{1}\right) \rightarrow B F\left(A_{1}^{\prime}\right)$ and $\eta: B F\left(A_{2}^{t}\right) \rightarrow B F\left(A_{2}^{\prime t}\right)$ such that $(\delta \otimes \eta)([X]) \equiv\left[X^{\prime}\right]$ in $B F\left(A_{1}^{\prime}\right) \otimes B F\left(A_{2}^{\prime t}\right)$ (see $[\mathrm{C} 1],[\mathrm{H}])$.

Theorem 2.2 (Cuntz [C1]). Let $A$ and $A^{\prime}$ be indecomposable $\{0,1\}$-matrices and have the forms of (2-2) with essentially irreducible nonpermutation diagonal blocks. Then $\overline{\mathcal{O}}_{A} \cong \overline{\mathcal{O}}_{A^{\prime}}$ implies $\mathcal{C}(A)=\mathcal{C}\left(A^{\prime}\right)$. In particular, $A \sim_{F E} A^{\prime}$ implies $\mathcal{C}(A)=$ $\mathcal{C}\left(A^{\prime}\right)$.

Remark 2.3. The invariant $\mathcal{C}(A)$ was derived in [C1] via $C^{*}$-algebra extensions ( $K K$-theory), in the case where the diagonal blocks $A_{1}$ and $A_{2}$ of $A$ are not essential permutation matrices. Given $A$ and $A^{\prime}$, for simplicity we suppose all the diagonal blocks are irreducible, there are short exact sequences $\rho: \overline{\mathcal{O}}_{A_{2}} \longmapsto \mathcal{O}_{A} \rightarrow \mathcal{O}_{A_{1}}$ and $\rho^{\prime}: \overline{\mathcal{O}}_{A_{2}^{\prime}} \longmapsto \mathcal{O}_{A^{\prime}} \rightarrow \mathcal{O}_{A_{1}^{\prime}}$. Cuntz showed that if $\overline{\mathcal{O}}_{A} \cong \overline{\mathcal{O}}_{A^{\prime}}$, then there is a producttype isomorphism $\beta: \operatorname{Ext}\left(\mathcal{O}_{A_{1}}, \mathcal{O}_{A_{2}}\right) \rightarrow \operatorname{Ext}\left(\mathcal{O}_{A_{1}^{\prime}}, \mathcal{O}_{A_{2}^{\prime}}\right)$ such that $\beta[\rho]=\left[\rho^{\prime}\right]$. So the pair $\left([\rho], \operatorname{Ext}\left(\mathcal{O}_{A_{1}}, \mathcal{O}_{A_{2}}\right)\right)$ is a stable isomorphism invariant. Cuntz also showed that $[\rho] \in \operatorname{Ext}\left(\mathcal{O}_{A_{1}}\right) \otimes K_{0}\left(\mathcal{O}_{A_{2}}\right) \subset \operatorname{Ext}\left(\mathcal{O}_{A_{1}}, \mathcal{O}_{A_{2}}\right)$ and the pair $([X] \cong[\rho]$, $\left.B F\left(A_{1}\right) \otimes B F\left(A_{2}^{t}\right) \cong \operatorname{Ext}\left(\mathcal{O}_{A_{1}}\right) \otimes K_{0}\left(\mathcal{O}_{A_{2}}\right)\right)$ is a stable isomorphism invariant for $\mathcal{O}_{A}$.

In $[\mathrm{H}]$, we reproved the Cuntz invariant of flow equivalence using matrix techniques from symbolic dynamics and showed the following.

Theorem 2.4 $([\mathrm{H}])$. Let $A$ and $A^{\prime}$ be as in Definition 2.1. Then $A \sim_{F E} A^{\prime}$ if and only if

(1) $A_{i} \sim_{F E} A_{i}^{\prime}, i=1,2$.

(2) $\mathcal{C}(A)=\mathcal{C}\left(A^{\prime}\right)$. 
If the irreducible components of $A$ and $A^{\prime}$ are not permutation matrices, then (1) can be replaced by $\left(1^{\prime}\right)$ : $\operatorname{sgn}\left(A_{i}\right)=\operatorname{sgn}\left(A_{i}^{\prime}\right), i=1,2$.

Now we are ready to show that $\mathcal{C}(A)$ alone is a complete stable isomorphism invariant for $\mathcal{O}_{A}$, as conjectured in $[\mathrm{H}]$.

To every matrix $A \in \mathrm{M}_{n}\left(\mathbb{Z}_{+}\right)$, we associate two matrices $A_{-} \in \mathrm{M}_{n+2}\left(\mathbb{Z}_{+}\right)$and $A_{\sim} \in \mathrm{M}_{n+3}\left(\mathbb{Z}_{+}\right)$given by

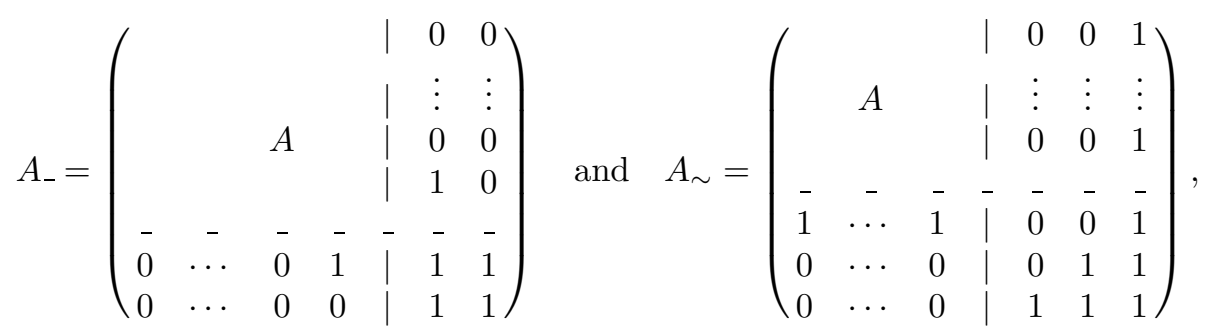

respectively $([\mathrm{C} 2],[\mathrm{R}])$. It is easy to check that $\operatorname{det}\left(I-A_{-}\right)=\operatorname{det}\left(I-A_{\sim}\right)=$ $-\operatorname{det}(I-A)$.

Lemma 2.5. Let $e_{1}, \ldots, e_{n}, e_{n+1}, e_{n+2}, e_{n+3}$ be the standard basis for $\mathbb{Z}^{n+3}$ (column space). Define

$$
\delta_{-}: B F(A) \rightarrow B F\left(A_{-}\right) \quad \text { by }\left[\sum_{i=1}^{n} \xi_{i} e_{i}\right] \mapsto\left[\sum_{i=1}^{n} \xi_{i} e_{i}+0 \cdot e_{n+1}+0 \cdot e_{n+2}\right]
$$

and

$\delta_{\sim}: B F(A) \rightarrow B F\left(A_{\sim}\right) \quad$ by $\left[\sum_{i=1}^{n} \xi_{i} e_{i}\right] \mapsto\left[\sum_{i=1}^{n} \xi_{i} e_{i}+0 \cdot e_{n+1}+0 \cdot e_{n+2}+0 \cdot e_{n+3}\right]$,

where $\xi_{i} \in \mathbb{Z}$. Then both $\delta_{-}$and $\delta_{\sim}$ are group isomorphisms. Similarly, we can define group isomorphisms

$$
\eta_{-}: B F\left(A^{t}\right) \rightarrow B F\left(A_{-}^{t}\right) \quad \text { and } \quad \eta_{\sim}: B F\left(A^{t}\right) \rightarrow B F\left(A_{\sim}^{t}\right) .
$$

Proof. Linear algebra.

Let $A=\left(\begin{array}{ll}1 & 1 \\ 1 & 1\end{array}\right)$; then

$$
A_{-}=\left(\begin{array}{llll}
1 & 1 & 0 & 0 \\
1 & 1 & 1 & 0 \\
0 & 1 & 1 & 1 \\
0 & 0 & 1 & 1
\end{array}\right)
$$

Denote by $\mathcal{O}_{2}$ and $\mathcal{O}_{2}$ the CK-algebras defined by the two matrices above. Cuntz showed in $[\mathrm{C} 2]$ that $\mathcal{O}_{2} \cong \mathcal{O}_{2_{-}}$implies $\overline{\mathcal{O}}_{A} \cong \overline{\mathcal{O}}_{A_{-}}$for any $\{0,1\}$-matrix $A$. This result of Cuntz, together with Franks' classification of irreducible topological Markov chains up to flow equivalence in $[\mathrm{F}]$, as well as Theorem 1.1, reduces the classification of simple CK-algebras up to stable isomorphism to the question: Is $\mathcal{O}_{2} \cong \mathcal{O}_{2}$ ? It has been open for many years until recently, Rørdam $[R]$ provides a positive answer.

The following is the main result of this note. 
Theorem 2.6. Let $A$ and $A^{\prime}$ be indecomposable $\{0,1\}$-matrices with two irreducible components as in Theorem 2.2. Then $\overline{\mathcal{O}}_{A} \cong \overline{\mathcal{O}}_{A^{\prime}}$ if and only if $\mathcal{C}(A)=\mathcal{C}\left(A^{\prime}\right)$, that is, they have the same Cuntz invariant as defined in Definition 2.1.

Proof. By Theorem 2.2, it suffices to prove the sufficiency.

Suppose $\mathcal{C}(A)=\mathcal{C}\left(A^{\prime}\right) \quad($ see Definition 2.1 and $(2-2))$. $\overline{\mathcal{O}}_{A^{\prime}}$.

If $\operatorname{sgn}\left(A_{i}\right)=\operatorname{sgn}\left(A_{i}^{\prime}\right), \quad i=1,2$, then by Theorems 1.1 and 2.4 , we have $\overline{\mathcal{O}}_{A} \cong$

Otherwise, suppose first that $\operatorname{sgn}\left(A_{1}\right)=-\operatorname{sgn}\left(A_{1}^{\prime}\right)$, and $A_{2}=A_{2}^{\prime}$. We shall derive a "diagonal block changing" method for CK-algebras, analogous to the Blocking Lemma for flow equivalence in $[\mathrm{H}]$.

Let

$$
A_{*}=\left(\begin{array}{ccc}
A_{1 *} & \mid & X_{*} \\
- & - & - \\
0 & \mid & A_{2}
\end{array}\right) \text {, where } A_{1 *}:=\left(A_{1}\right)_{\sim}, X_{*}:=\left(\begin{array}{ccc}
X & \\
- & - & - \\
0 & \cdots & 0 \\
0 & \cdots & 0 \\
0 & \cdots & 0
\end{array}\right) .
$$

Let

$$
A_{* *}=\left(\begin{array}{ccc}
A_{1 * *} & \mid & X_{* *} \\
- & - & - \\
0 & \mid & A_{2}
\end{array}\right), \quad \text { where } \quad A_{1 * *}:=\left(A_{1 *}\right)_{-}, \quad X_{* *}:=\left(\begin{array}{ccc}
X_{*} & \\
- & - & - \\
0 & \cdots & 0 \\
0 & \cdots & 0
\end{array}\right) .
$$

By Lemma 2.5, it is easily seen that

$$
\mathcal{C}(A)=\mathcal{C}\left(A_{*}\right)=\mathcal{C}\left(A_{* *}\right) .
$$

Note that $\operatorname{det}\left(I-A_{1}\right)=\operatorname{det}\left(I-A_{1 * *}\right)$ and $\operatorname{sgn}\left(A_{1}^{\prime}\right)=\operatorname{sgn}\left(A_{1 *}\right)$. By Theorems 1.1 and 2.4 , we have $\overline{\mathcal{O}}_{A} \cong \overline{\mathcal{O}}_{A_{* *}}$, and $\overline{\mathcal{O}}_{A^{\prime}} \cong \overline{\mathcal{O}}_{A_{*}}$. Thus in order to show that $\overline{\mathcal{O}}_{A} \cong \overline{\mathcal{O}}_{A^{\prime}}$, it suffices to show that $\mathcal{O}_{A_{*}} \cong \mathcal{O}_{A_{* *}}$. Here we need a trick of Cuntz [C2] to show that $\mathcal{O}_{2} \cong \mathcal{O}_{2_{-}}$implies $\overline{\mathcal{O}}_{A} \cong \overline{\mathcal{O}}_{A_{-}}$, which was elaborated in the Appendix of $[R]$. It turns out that the argument there for simple CK-algebras can be adapted for two-component CK-algebras as follows.

Since $\mathcal{O}_{2} \cong \mathcal{O}_{2_{-}}(\operatorname{Røddam}[\mathrm{R}])$, as was shown in the Appendix, there is a $C^{*}$ algebra

$$
\mathcal{E}=C^{*}\left(s_{1}, s_{2}, e\right)=C^{*}\left(t_{1}, t_{2}, t_{3}, t_{4}, e\right)
$$

with unit 1 , where $e$ is a one-dimension projection, $s_{i}$ 's and $t_{j}$ 's are partial isometries satisfying

$$
\begin{aligned}
1=s_{1} s_{1}^{*}+s_{2} s_{2}^{*}, \quad s_{1}^{*} s_{1}=s_{2}^{*} s_{2}=1 \\
1=t_{1} t_{1}^{*}+t_{2} t_{2}^{*}+t_{3} t_{3}^{*}+t_{4} t_{4}^{*}, \\
t_{1}^{*} t_{1}=t_{1} t_{1}^{*}+t_{2} t_{2}^{*} \\
t_{2}^{*} t_{2}=t_{1} t_{1}^{*}+t_{2} t_{2}^{*}+t_{3} t_{3}^{*} \\
t_{3}^{*} t_{3}=t_{2} t_{2}^{*}+t_{3} t_{3}^{*}+t_{4} t_{4}^{*} \\
t_{4}^{*} t_{4}=t_{3} t_{3}^{*}+t_{4} t_{4}^{*}
\end{aligned}
$$

and $e s_{1}=s_{1} e=e, e t_{1}=t_{1} e=e$. 
Suppose the sizes of $A_{1}$ and $A_{2}$ are $n$ and $m$, respectively. Let $\mathfrak{A}$ be the algebra $\mathbb{C} \oplus \cdots \oplus \mathbb{C} \oplus \mathcal{E}$, with $n+m$ copies of $\mathbb{C}$, and denote by $f_{1}, \ldots, f_{n}$ and $g_{1}, \ldots, g_{m}$ the one-dimension projections onto each of these $n+m$ copies. Represent $\mathfrak{A}$ on a Hilbert space $H$ such that every nonzero projection in $\mathfrak{A}$ becomes infinite on $H$.

Then find partial isometries $x_{1}, \ldots, x_{n+1}$ on $H$ satisfying

$$
x_{i} x_{i}^{*}=f_{i}, \quad x_{i}^{*} x_{i}=s_{2} s_{2}^{*}+\sum_{j=1}^{n} A_{1}(i, j) f_{j}+\sum_{j=1}^{m} X(i, j) g_{j},
$$

for $i=1, \ldots n$ and

$$
x_{n+1} x_{n+1}^{*}=e, \quad x_{n+1}^{*} x_{n+1}=s_{2} s_{2}^{*}+f_{1}+\cdots+f_{n} .
$$

Let $x_{n+2}=s_{1}(1-e)$ and $x_{n+3}=s_{2}$. Then find partial isometries $\hat{x}_{1}, \ldots, \hat{x}_{m}$ on $H$ satisfying

$$
\hat{x}_{i} \hat{x}_{i}^{*}=g_{i}, \quad \hat{x}_{i}^{*} \hat{x}_{i}=\sum_{j=1}^{m} A_{2}(i, j) g_{j},
$$

for $i=1, \ldots, m$. Let $x_{n+3+i}=\hat{x}_{i}, i=1, \ldots, m$. Then it is easy to check as in $[\mathrm{R}]$ that the $C^{*}$-algebra $\mathfrak{B}_{1}$ generated by $x_{1}, \ldots, x_{n+m+3}$ contains $\mathfrak{A}$ and is isomorphic to $\mathcal{O}_{A_{*}}$.

Next, find partial isometries $u_{1}, \ldots, u_{n+1}$ in $\mathfrak{A}$ so that $u_{i} u_{i}^{*}=x_{i}^{*} x_{i}$, and

$$
\begin{gathered}
u_{i}^{*} u_{i}=t_{2} t_{2}^{*}+\sum_{j=1}^{n} A_{1}(i, j) f_{j}+\sum_{j=1}^{m} X(i, j) g_{j} \quad(i=1, \ldots, n), \\
u_{n+1}^{*} u_{n+1}=t_{2} t_{2}^{*}+f_{1}+\cdots+f_{n} .
\end{gathered}
$$

Such $u_{i}$ 's exist because $s_{2} s_{2}^{*}$ is equivalent to $t_{2} t_{2}^{*}$ in $\mathcal{E}$ due to $(2-3)$ and (2-4). Set $y_{i}=x_{i} u_{i}$ (hence $\left.x_{i}=y_{i} u_{i}^{*}\right)$ for $i=1, \ldots, n+1, y_{n+2}=t_{1}(1-e), y_{n+3}=t_{2}, y_{n+4}=$ $t_{3}, \quad y_{n+5}=t_{4}$ and $y_{n+5+i}=\hat{x}_{i}, i=1, \ldots, m$. Again, we can check that the $C^{*}$ algebra $\mathfrak{B}_{2}$ generated by $y_{1}, \ldots, y_{n+m+5}$ contains $\mathfrak{A}$ and hence is equal to $\mathfrak{B}_{1}$. But $\mathfrak{B}_{2} \cong \mathcal{O}_{A_{* *}}$, therefore $\mathcal{O}_{A_{*}} \cong \mathcal{O}_{A_{* *}}$.

Now consider $A_{1}=A_{1}^{\prime}$ but $\operatorname{sgn}\left(A_{2}\right)=-\operatorname{sgn}\left(A_{2}^{\prime}\right)$. We can change $A_{2}$ into $A_{2}^{\prime}$ by a stable isomorphism of $\mathcal{O}_{A}$, with a possible change in the off-diagonal block. The method used to do this is similar to that above, where $X_{*}$ and $X_{* *}$ will be defined correspondingly as matrices obtained from $X$ by adding some zero columns to its right. Therefore, it remains to show that $\overline{\mathcal{O}}_{A} \cong \overline{\mathcal{O}}_{A^{\prime}}$ when they have the same Cuntz invariant and flow equivalent diagonal blocks of their defining matrices. But this follows from Theorems 1.1 and 2.4.

Remark 2.7. In the proof of Theorem 2.6, we actually have encountered a CKsubalgebra defined by a degenerate $\{0,1\}$-matrix, since $A_{1}$ and $A_{2}$ are generally essentially irreducible. Being merely essentially irreducible, $A_{1}$ and $A_{2}$ may not be uniquely defined to make $A$ a $2 \times 2$ block upper-triangular matrix. In [C2], $A_{1}$ is chosen to be irreducible, while $A_{2}$ generally have zero columns and is called the saturation of its irreducible core matrix. However, given a matrix $A$ with its maximal nondegenerate principal submatrix $A_{\text {core }}$, one can see that $\mathcal{O}_{A} \cong \mathcal{O}_{A_{\text {core }}}$ and $K_{0}\left(\mathcal{O}_{A}\right) \cong K_{0}\left(\mathcal{O}_{A_{\text {core }}}\right)$ canonically. Also as was shown in $[\mathrm{H}]$, the Cuntz 
invariant is independent of possible choices of $A_{1}$ and $A_{2}$ for a $2 \times 2$ block uppertriangular matrix $A$. In fact, in order to compute Cuntz invariant, we can change all essentially irreducible diagonal block matrices into their irreducible cores by flow equivalence (but then the resulting matrix might be over $\mathbb{Z}_{+}$instead of over $\{0,1\}$, see $[\mathrm{H}])$. We have seen that Cuntz invariant is a perfect stable isomorphism invariant for two-component CK-algebras. However, it is still not clear how one could classify these algebras up to unital isomorphism.

The Cuntz invariant is rather computable. In some special cases, the invariant has an even simpler equivalent version. Here is an example, where all the CKalgebras associated to diagonal block matrices of $A$ are stably isomorphic to the Cuntz algebra $\mathcal{O}_{n}(n \geq 2)$.

Corollary 2.8. Let $A$ and $A^{\prime}$ be as in Theorem 2.6. Suppose that $K_{0}\left(\mathcal{O}_{A_{i}}\right)$ is a finite cyclic group, $i=1,2$. Then $\overline{\mathcal{O}}_{A} \cong \overline{\mathcal{O}}_{A^{\prime}}$ if and only if $K_{0}\left(\mathcal{O}_{A_{i}}\right) \cong K_{0}\left(\mathcal{O}_{A_{i}^{\prime}}\right)$, $i=1,2$, and $K_{0}\left(\mathcal{O}_{A}\right) \cong K_{0}\left(\mathcal{O}_{A^{\prime}}\right)$.

In particular, if $A_{1}\left(A_{1}^{\prime}\right)$ is an $n \times n\left(n^{\prime} \times n^{\prime}\right)$ matrix with all entries being 1 and $A_{2}\left(A_{2}^{\prime}\right)$ is an $m \times m\left(m^{\prime} \times m^{\prime}\right)$ matrix also with all entries being 1 , where $n, n^{\prime}, m, m^{\prime} \geq 2$, and $X$ and $X^{\prime}$ are not a zero matrix, then $\overline{\mathcal{O}}_{A} \cong \overline{\mathcal{O}}_{A^{\prime}}$ if and only if $n=n^{\prime}, m=m^{\prime}$ and $\operatorname{gcd}\{n-1, m-1, l\}=\operatorname{gcd}\left\{n^{\prime}-1, m^{\prime}-1, l^{\prime}\right\}$, where $l=\sum_{i, j} X(i, j)$ and $l^{\prime}=\sum_{i, j} X^{\prime}(i, j)$.

Proof. It suffices to prove the sufficiency. For the first part, as in the proof Theorem 2.6 , we can assume that $\operatorname{sgn}\left(A_{i}\right)=\operatorname{sgn}\left(A_{i}^{\prime}\right)=-, i=1,2$. Notice that $K_{0}\left(\mathcal{O}_{A}\right) \cong$ $K_{0}\left(\mathcal{O}_{A^{\prime}}\right)$ if and only if $B F(A) \cong B F\left(A^{\prime}\right)$. Thus we have $A_{i} \sim_{F E} A_{i}^{\prime}, \quad i=1,2$. It then follows from Proposition 3.7 of $[\mathrm{H}]$ that $A \sim_{F E} A^{\prime}$, and by Theorem 1.1 we have $\overline{\mathcal{O}}_{A} \cong \overline{\mathcal{O}}_{A^{\prime}}$.

For the second part of the corollary, it suffices to show that $A \sim_{F E} A^{\prime}$. Using the inverse operation of state splitting for topological Markov chains (see [F], where a state splitting is given explicitly by a splitting of the $i$-th row (column) and a replicating of the $i$-th column (row) of the adjacency matrix; also cf. [Bo]), we can see that $A$ is strongly shift equivalent to $\left(\begin{array}{ll}n & l \\ 0 & m\end{array}\right)$, and $A^{\prime}$ is strongly shift equivalent to $\left(\begin{array}{cc}n^{\prime} & l^{\prime} \\ 0 & m^{\prime}\end{array}\right)$. Note that strong shift equivalence implies flow equivalence and that the Cuntz invariants are preserved during these moves of flow equivalence. The rest then again follows from Proposition 3.7 of $[\mathrm{H}]$.

\section{ACKNOWLEDGMENT}

The author gratefully acknowledges very useful correspondence with J. Cuntz and M. Rørdam. Also he would like to thank the referee for the kind suggestions.

\section{REFERENCES}

[Bo] M. Boyle, Symbolic dynamics and matrices, Combinatorial and Graph-Theoretic Problems in Linear Algebra, IMA Volumes in Math and Appl. Vol. 50 (R. Brualdi, S. Friedland, and V. Klee, eds.), Springer-Verlag, New York 1993, pp. 1-38. MR 94g:58062

[BF] R. Bowen and J. Franks, Homology for zero-dimensional basic sets, Ann. of Math. (2) 106 (1977), 73-92. MR 56:16692

[C1] J. Cuntz, A class of $C^{*}$-algebras and topological Markov chains II: reducible chains and the Ext-functor for $C^{*}$-algebras, Inventiones Math. 63 (1981), 25-40. MR 82f:46073b

[C2] The classification problem for the $C^{*}$-algebras $\mathcal{O}_{A}$, Geometric methods in operator algebras (H. Araki and E. G. Effros, eds.), Longman, New York, 1986, pp. 145-151. MR 88a:46081 
[C3] - Personal communication.

[C4] - On the homotopy groups of endomorphisms of a $C^{*}$-algebra (with applications to topological Markov chains), Proc. of O.A.G.R. Conference in Neptun, Romania, Pitman, 1984, pp. 124-137. MR 86a:46093

[CK] J. Cuntz and W. Krieger, A class of $C^{*}$-Algebras and topological Markov chains, Inventiones Math. 56 (1980), 251-268. MR 82f:46073a

[F] J. Franks, Flow equivalence of subshifts of finite type, Ergodic Theory Dynamical Systems 4 (1984), 53-66. MR 86j:58078

$[\mathrm{H}] \quad$ D. Huang, Flow equivalence of reducible shifts of finite type, Ergodic Theory Dynamical Systems 14 (1994), 695-720. CMP 95:04

[PS] W. Parry and D. Sullivan, A topological invariant for flows on one dimensional spaces, Topology 14 (1975), 297-299.

[R] M. Rørdam, Classification of Cuntz-Krieger algebras, K-theory 9 (1995), 31-58.

Department of Mathematics, University of Washington, Box 354350, Seattle, WashINGTON 98195-4350

E-mail address: dhuang@math.washington.edu 